\title{
Understanding the concurrent microstructural evolution and its impact on superplastic characteristics: an overview
}

\author{
B. P. Kashyap ${ }^{\dagger}$ \\ †bpk@iitj.ac.in \\ Department of Metallurgical and Materials Engineering, Indian Institute of Technology Jodhpur, \\ NH 65 Nagaur Road, Karwar-342037, Rajasthan, India
}

\begin{abstract}
Extensive studies on experimental constitutive relationship for superplastic deformation of both the ideal equiaxed microstructure and the non-equiaxed microstructure were taken up over a period of more than four decades in the group. While our understanding of microstructure-superplastic flow relationship has grown significantly, there remains a gap between the theoretical modelling, based on steady-state, and the realistic behavior investigated for several materials. The observations made here are also supported by numerous publications by other investigators in the literature, with growing development of not only metals but ceramics, composites, intermetallic compounds and nano-crystalline materials aimed to push superplastic behavior to higher strain rate and lower temperature condition. Here, an attempt is made to list the range of microstructure changes that occur and as such the need to consider the same while evaluating the parameters of constitutive relationship. Some suggestions are provided to minimize the gap between the ideal and real behavior of superplastic flow based on the types of stress-strain curves reported in the superplastic literature and their effect on the nature of stress-strain rate relation. It includes the consideration of effective stress for superplastic deformation, and to incorporate the effects of strain and concurrent microstructure in the experimental constitutive relationship.
\end{abstract}

Keywords: constitutive relationship, effective stress, microstructure evolution, mechanism for superplastic deformation, pseudo-steady state.

\section{Introduction}

Owing to the industrial applications and a wide range of micro-mechanisms involved in understanding the phenomena of superplasticity, there remain several challenges and problems to be resolved, in spite of a rapid progress made over last eight decades. One of the problems involves developing universal constitutive relationship for superplasticity and its verification through experimental parameters and concurrent microstructural evolution that leads to the deviation from the requirement of true steady state condition. Firstly, in the modeling of superplasticity, it is invariably considered that the microstructure consists of fine equiaxed grains, which remain stable in the course of superplastic deformation due to grain boundary sliding and its accommodation by grain rotation, diffusion and directed dislocation slip. However, there exist ample evidences to support that the fine equiaxed grains undergo substantial grain growth during superplastic deformation due to diffusion assisted grain boundary migration occurring for accommodation of grain boundary sliding. In the absence of adequate accommodation of grain boundary sliding there also occurs cavitation [1]. Such microstructural changes influence the nature of stress-strain curves in the form of flow hardening, flow softening or their equal and opposite effects that can lead to pseudo-steady state condition in contrast to what is assumed in superplastic deformation. Secondly, the large number of superplastic materials including metals, ceramics, intermetallic compounds and composites do not meet the condition of equiaxed grains. Instead the initial grains in as-processed or as-worked state are reported to be elongated or banded $[2,3]$. Such microstructures are reported to be highly unstable and undergo morphological as well as size changes during the early part of deformation at elevated temperatures. Accordingly, these changes also contribute to strain sensitive flow stress during superplastic deformation. Therefore, the present work aims to critically bring the sources of microstructure evolution and the methods that can minimize the deviation from steady state. Alternatively, one can incorporate the effects of strain and/or changes in microstructure towards modifying the constitutive relationship for superplasticity.

\section{Constitutive relationship for superplastic deformation}

The phenomena of superplasticity, exhibiting exceptionally large ductility of several hundred percent, with the world record being $8000 \%$ in the aluminum bronze [4], occurs in certain materials having fine grains $(\leq 10 \mu \mathrm{m}$ for metals and $\leq 1 \mu \mathrm{m}$ for ceramics) and at the test conditions of intermediate strain rates $\left(\sim 10^{-5}-10^{-2} \mathrm{~s}^{-1}\right)$ and high temperature $\left(\geq 0.5 T_{\mathrm{m}}\right.$, where $T_{\mathrm{m}}$ is absolute melting point). Several mechanisms for superplastic deformation have been proposed in the literature [4] to suggest the importance of grain boundary sliding as well as the stability of starting fine equiaxed microstructure. 
Tensile ductility during superplastic deformation is closely related to the value of strain rate sensitivity index $m$, which should be large $(m \geq 0.3)$, as it avoids neck formation and/or its propagation. This $m$ is an important parameter, which is nothing but an inverse of stress exponent $n(m=1 / n)$ of the constitutive relationships for creep and high temperature deformation. The effects of temperature, strain rate and grain size for superplastic behavior are thus conveniently derived from these equations with the highlights brought by the presence of fine grains in terms of grain boundary sliding exhibiting a positive role in deformation. The various forms of constitutive relations [4] and the models [5] are elegantly summarized in some review papers [4,5]. The creep equation which is commonly used for evaluating the parameters of the constitutive relationship for superplastic deformation is of type $[6,7]$

$$
\begin{gathered}
\dot{\varepsilon}=\frac{A D_{0} G b}{k T}\left(\frac{b}{d}\right)^{p}\left(\frac{\sigma}{G}\right)^{n=\frac{1}{m}} \exp \left(-\frac{Q}{R T}\right) \\
\dot{\varepsilon}=\frac{A D_{0} G b}{k T}\left(\frac{b}{d}\right)^{p}\left(\frac{\sigma_{e}=\sigma-\sigma_{T H}-\sigma_{i}}{G}\right)^{n=\frac{1}{m}} \exp \left(-\frac{Q}{R T}\right),
\end{gathered}
$$

where $A, b, \sigma, k, p, n, d$ and $G$ are material constants, magnitude of Burgers vector, flow stress, Boltzmann's constant, inverse grain size exponent, stress exponent, grain size and Young's modulus, respectively, $\dot{\varepsilon}$ is strain rate, $D_{0} \exp (-Q / R T)$ is diffusion coefficient $(D)$ with $D_{0}$ being the attempt frequency, $R$ the universal gas constant, $Q$ the activation energy for diffusion and $T$ is absolute temperature. The parameters like $n$ and $Q$ of constitutive relationship are noted to have exceptionally large values which cannot be explained with the help of basic mechanisms or theories for high temperature phenomena. In such cases, plastic deformation is related to the effective stress $\left(\sigma_{e}\right)$, which is the difference between applied stress $(\sigma)$ and threshold stress $\left(\sigma_{T H}\right)$ and/or internal stress $\left(\sigma_{i}\right)$ [7]. The magnitudes of $\sigma_{T H}$ and $\sigma_{i}$ constitute a large fraction of applied stress and this resistance to deformation is caused by dispersion of particles and/or concurrent development of dislocation structure. Fig. 1 shows the variations in applied stress, internal stress and effective stress as a function of strain rate as measured by stress dip test technique [8]. Also included in the figure is the plot of strain rate $\dot{\varepsilon}_{r 0}$, as obtained at the onset of stress relaxation curve employed in the stress-dip test for internal stress measurement. The ratio of internal stress to applied stress $\left(\sigma_{i} / \sigma\right)$ was reported to vary with test temperature and strain rate in the $\mathrm{Pb}-\mathrm{Sn}$ eutectic alloy. For example, the fraction of $\sigma_{i} / \sigma$ varied from about $60 \%$ to $73 \%$ depending on test temperature during superplastic deformation. However, the grain size was shown not to reveal any effect on this ratio.

\section{Types of as-worked microstructures employed in superplastic studies}

Although the standard microstructure for superplasticity is known to be the fine equiaxed grains the equally known in the literature are the as-worked microstructures of a wide variation in morphology [2,3]. Shown in Fig. 2 are some typical examples of the range of microstructures which have been investigated for superplastic studies. These microstructures are the result of mechanical or thermomechanical processing employed for getting superplasticity in commercial grade of standard or newly developed alloys.

The micrographs in Fig. 2 mostly represent the microstructures in two (for rod) or three (for sheet) mutually perpendicular surfaces. Usually, it requires a well-designed processing sequence or several trial and error approaches to get fine equiaxed grains like the one illustrated in Fig. 2a [9]. The microstructures obtained by rolling, extrusion or swaging of rod are highly elongated in the longitudinal direction of the worked product, Fig. 2b, whereas the transverse section shows equiaxed grains, Fig. 2c. Such microstructures retain their morphology during static annealing even though the grains grow with time and temperature. The experience in the $\mathrm{Pb}-\mathrm{Sn}$ eutectic alloy revealed this to be the case even after about nine months of annealing at as high a temperature as $0.97 T_{\mathrm{m}}$ (annealing temperature of $170^{\circ} \mathrm{C}$ and melting point of $183^{\circ} \mathrm{C}$ ). The sheets obtained by hot rolling or thermomechanical processing of plate exhibit either elongated $[11,12]$ or banded structure [13]. Fig. 2 d shows pancake shape in a superplastic $8090 \mathrm{Al}-\mathrm{Li}$ alloy [11] with plate like planarlinear orientation having elongated grains in the longitudinal and transverse sections but the rolling surface exhibiting equiaxed grains. A similar microstructure was also reported in 25.7Cr-6.6Ni microduplex stainless steel [12]. Also noted in superplastic $8090 \mathrm{Al}-\mathrm{Li}$ alloy are the differences in grain sizes and micro-textures between the surface layer (marked by $\mathrm{A}$ in Fig. 2d) as shown in Fig. 2e and the mid-thickness layer (marked by B in Fig. 2d) as shown in Fig. 2f, even in a thin sheet of $1.8 \mathrm{~mm}$ thickness. Such a behavior is ascribed to the presence of fine precipitates/particles like $\mathrm{Al}_{3} \mathrm{Zr}$, which get migrated towards the core of the ingot during the solidification process. The banded structure evolved during hot rolling, Fig. $2 \mathrm{~g}$, can contain aggregates of dynamically recrystallized very fine equiaxed grains in the direction of rolling, as illustrated through the high magnification image of the rolled surface of IN718 superalloy, Fig. 2h [13].

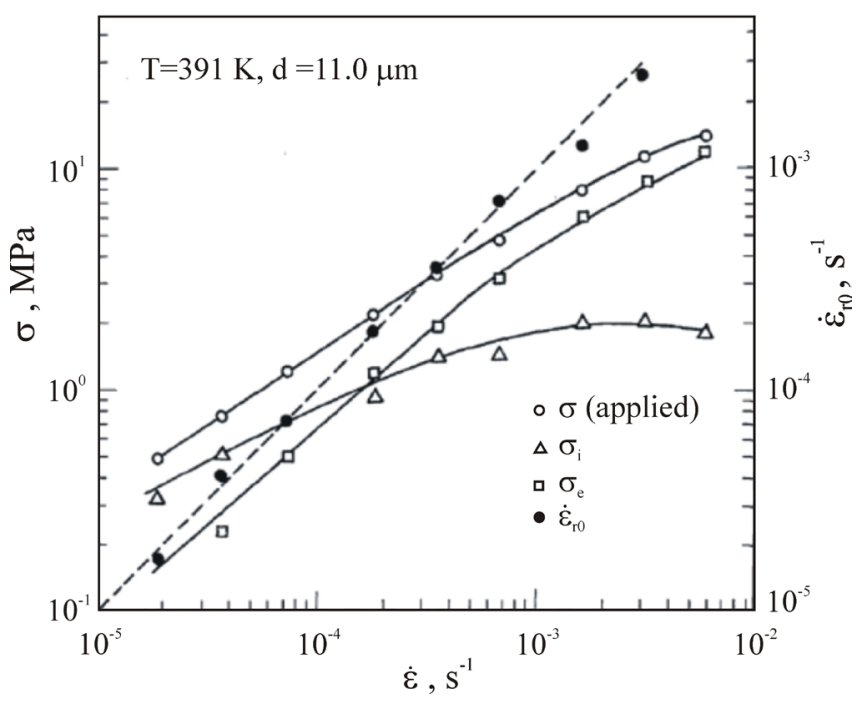

Fig. 1. Log-log plot of applied stress $(\sigma)$, internal stress $\left(\sigma_{i}\right)$ and effective stress $\left(\sigma_{e}\right)$ as a function of applied strain rate $(\dot{\varepsilon})$ in a $\mathrm{Pb}-\mathrm{Sn}$ eutectic alloy along with the plot of strain rate calculated at the onset (zero time) of stress relaxation curve $\left(\dot{\varepsilon}_{r 0}\right)[8]$. 


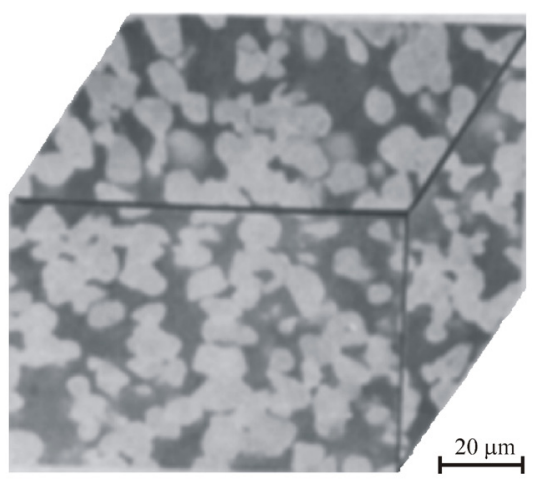

a

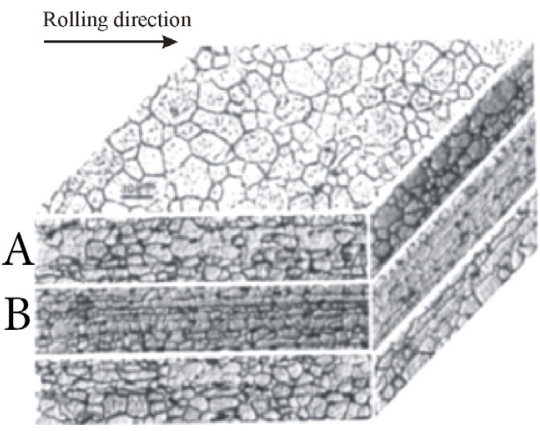

d

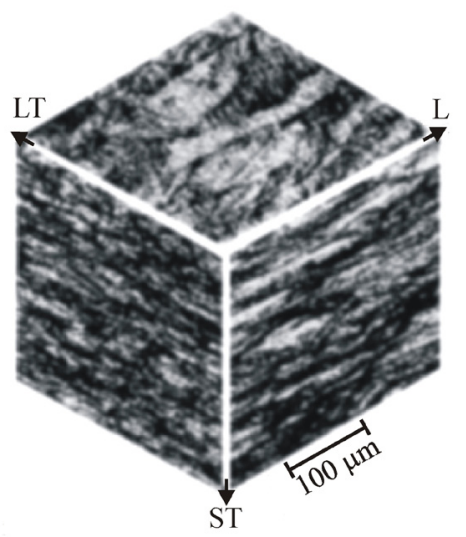

g

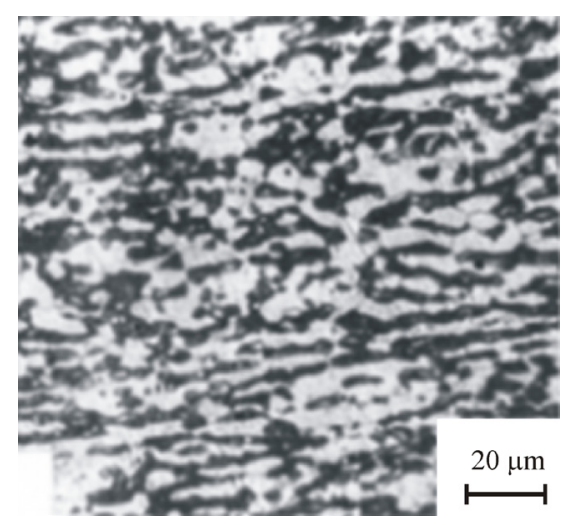

b

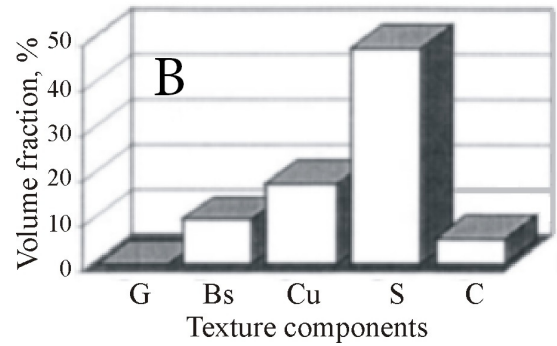

e

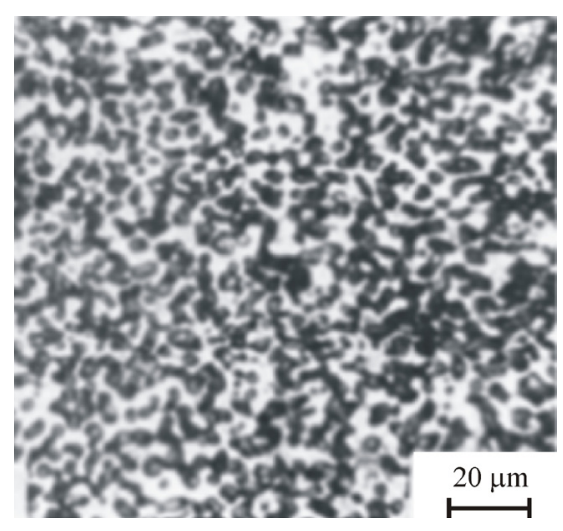

c

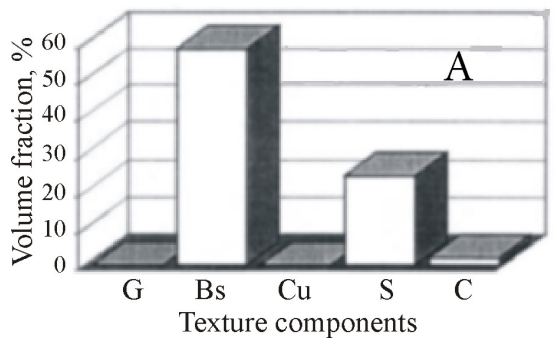

f

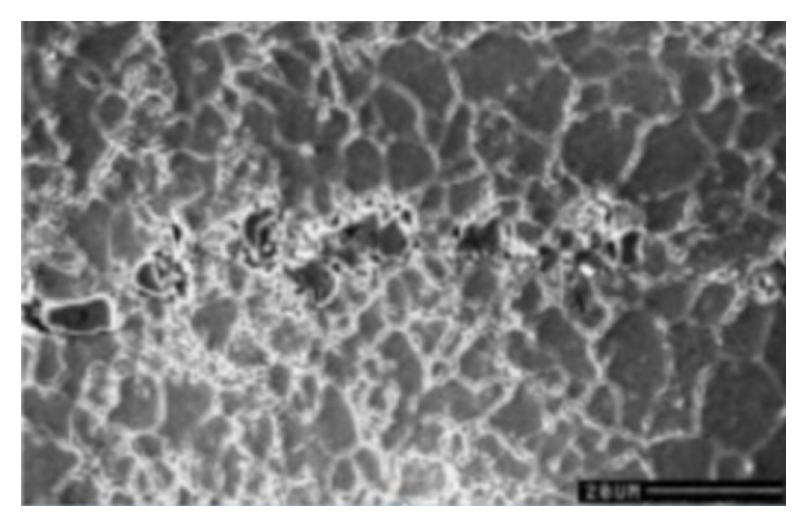

$\mathrm{h}$

Fig. 2. Various kinds of initial microstructures employed in superplastic studies: equiaxed grains in Al-Cu eutectic [9] (a); elongated grains (linear orientation-longitudinal elongated (b) and equiaxed transverse (c) grains) in Pb-Sn eutectic [10]; microstructure, plate like planarlinear orientation- equiaxed grains in rolling plane and elongated grains in transverse section (d), and micro-texture (e,f) variations along thickness between center (layer B) and surface (layer A) in Fig. 2d of rolled 8090 Al-Li alloy [11], similar to that (not shown here) in $25.7 \mathrm{Cr}-6.6 \mathrm{Ni}$ microduplex stainless steel [12]; banded microstructure in IN718 superalloy (g) with the bands of fine equiaxed grains revealed at high magnification [13] (h).

\section{Types of variation in flow stress with strain}

While the stress-strain curves to represent the steady state behavior needs to exhibit constant flow stress with increasing strain, in reality, it is very common that one finds the flow stress to vary with strain. The nature of variation in stressstrain curve depends on initial microstructure, and the test conditions like strain rate and temperature. In addition, some artificial effects of strain rate used to appear in the absence of true constant strain rate type tests. Such artifacts of results are eliminated easily these days by making use of the computerized system to renew the speed of the actuator or cross-head of the universal testing machine with the progress in straining. Some typical examples of stress-strain curves in superplastic literature are presented in Fig. 3.

The stress-strain curves for Al-Cu eutectic alloy [9], having fine equiaxed grains and given at a constant strain rate of $10^{-4} \mathrm{~s}^{-1}$ over the temperature range of $400-540^{\circ} \mathrm{C}$ in Fig. 3a, shows the effect of test temperature. While at lower test temperature there occurs flow hardening in the early part of deformation, the same approaches a pseudo-steady state at the later part of deformation. At higher test temperatures, however, the pseudo-steady state behavior is established right from the beginning of plastic deformation. The strain rate was shown to have similar effects but the pseudo-steady state behavior getting established from the beginning at lower 


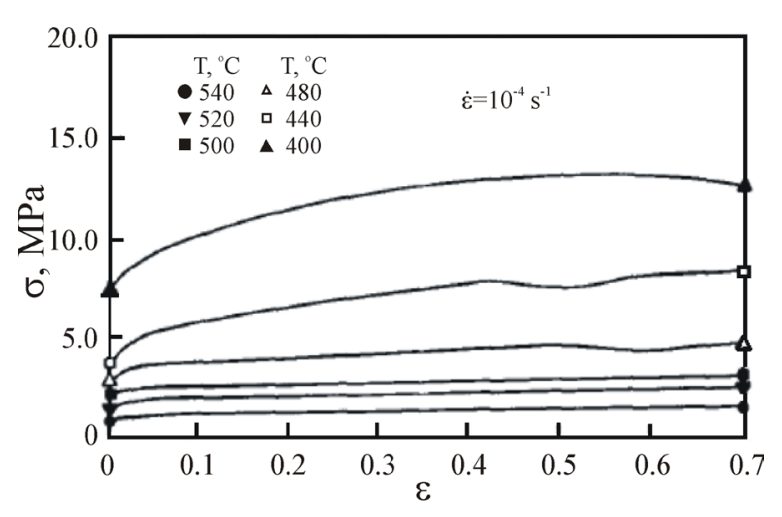

a

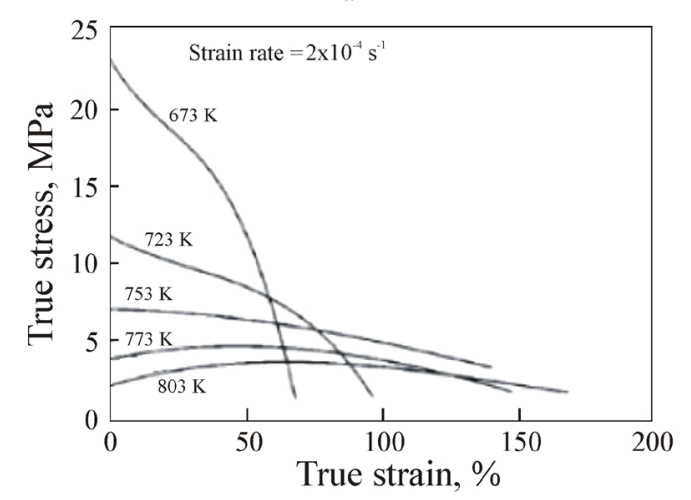

c

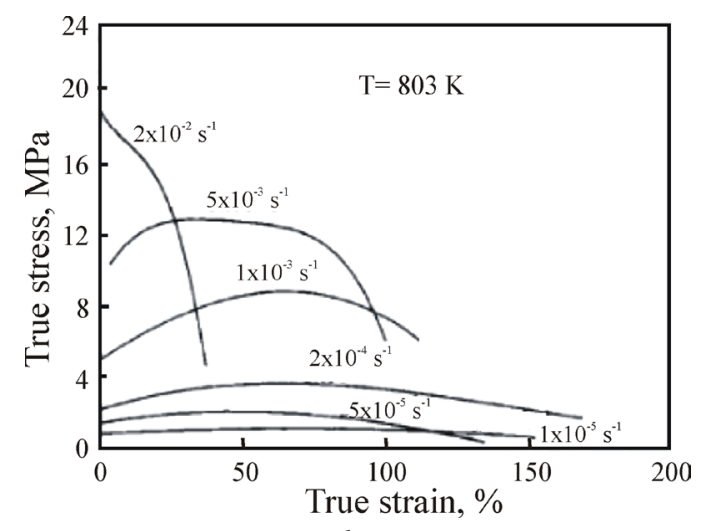

b

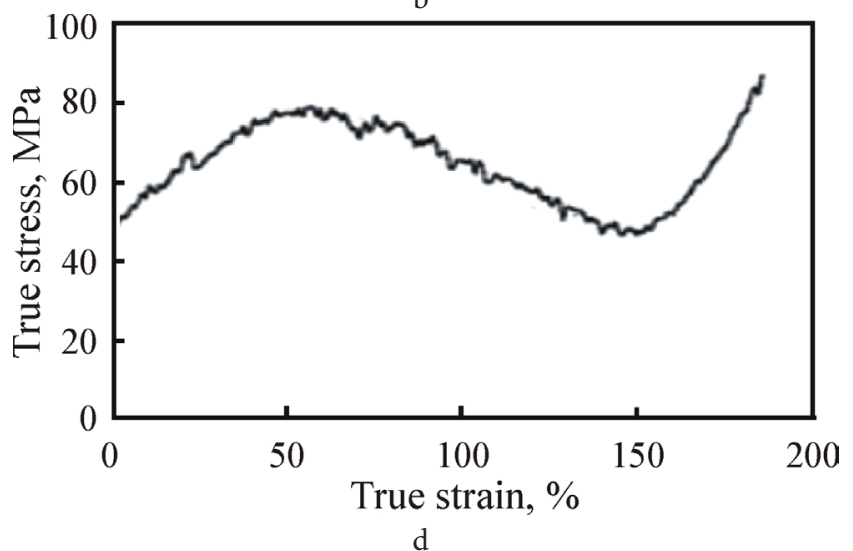

Fig. 3. Varying nature of stress-strain curves exhibited by superplastic materials: Al-Cu eutectic having equiaxed grains [9] (a), Al-Li alloy having pancake shaped grains [14] (b, c), IN 718 superalloy having banded structure $[13,15](d)$.

strain rates whereas the sequence of transient flow hardening followed by pseudo-steady state behavior occurring at higher strain rates. The fine grained but banded and/or elongated microstructures do not favor the steady state condition in general, except if there occur equal and opposite effects of different types of microstructure changes. For example, the stress-strain curve given for $8090 \mathrm{Al}-\mathrm{Li}$ alloy [14] in Fig. 3b at $803 \mathrm{~K}$ and strain rate of $10^{-5} \mathrm{~s}^{-1}$ exhibits pseudo steady state. Fig. 3b,c reveals flow hardening, flow softening and a combination of the two to varying extent depending on the strain rate and test temperature condition. The range of microstructure changes that occur during superplastic deformation may also exhibit flow hardening followed by flow softening and then again the flow hardening effect as illustrated in Fig. 3d for IN718 super-alloy having banded microstructure initially and undergoing grain growth, grain redistribution and cavitation $[13,15]$.

\section{Deviation from steady state (microstructure and flow stress)}

Steady state during superplastic deformation is considered to be satisfied when (i) the microstructure is stable, which appears in terms of grain size in the constitutive relationship, Eq. (1); (ii) the stress-strain rate relationship is valid with no variation in stress as a function of strain. Shown in Fig. 4 are some typical examples to illustrate the fact that one or both of the above conditions are not satisfied. Such deviations from steady state condition are widely reported in the literature [2,3,9-17]. Fig. 4a shows that the initially fine elongated grains become coarser with a simultaneous tendency of becoming equiaxed grains [10]. While grain growth leads to flow hardening, the change from elongated to equiaxed shapes of grains causes flow softening. Interestingly, there appear equal but opposite effects of these two types of microstructure changes in the $\mathrm{Pb}-\mathrm{Sn}$ eutectic, which results in stress-strain rate relationship that is independent of strain involved in differential strain rate (strain rate change) cycles from lower strain rate to higher strain rate repeatedly for four times. A similar change of concurrent grain growth and elongated grains becoming equiaxed grains, corresponding to the stress-strain curve of 8090 Al-Li alloy given in Fig. 4b, is presented as a function of strain in Fig. 4c. Also shown along with this are the texture evolution towards randomization, and the change from subgrains or low angle grain boundaries to grains or high angle grain boundaries, Fig. 4d [16]. In this case of a simultaneous decrease in grain elongation, grain growth, randomization of texture and increase in high angle grain boundaries, the flow stress was found to increase with strain initially then decrease with further straining, Fig. $4 \mathrm{a}$. The flow hardening in stress-strain curves observed during superplastic deformation is ascribed to concurrent grain growth. However, the analysis of microstructure and nature of stress-strain curves in several cases suggest that while this is true at lower strain rate and higher temperature conditions, the grain growth per se is not sufficient to quantitatively account for flow hardening [9]. Instead, a part of flow hardening, even in superplastic condition, arises through conventional work hardening accompanied 

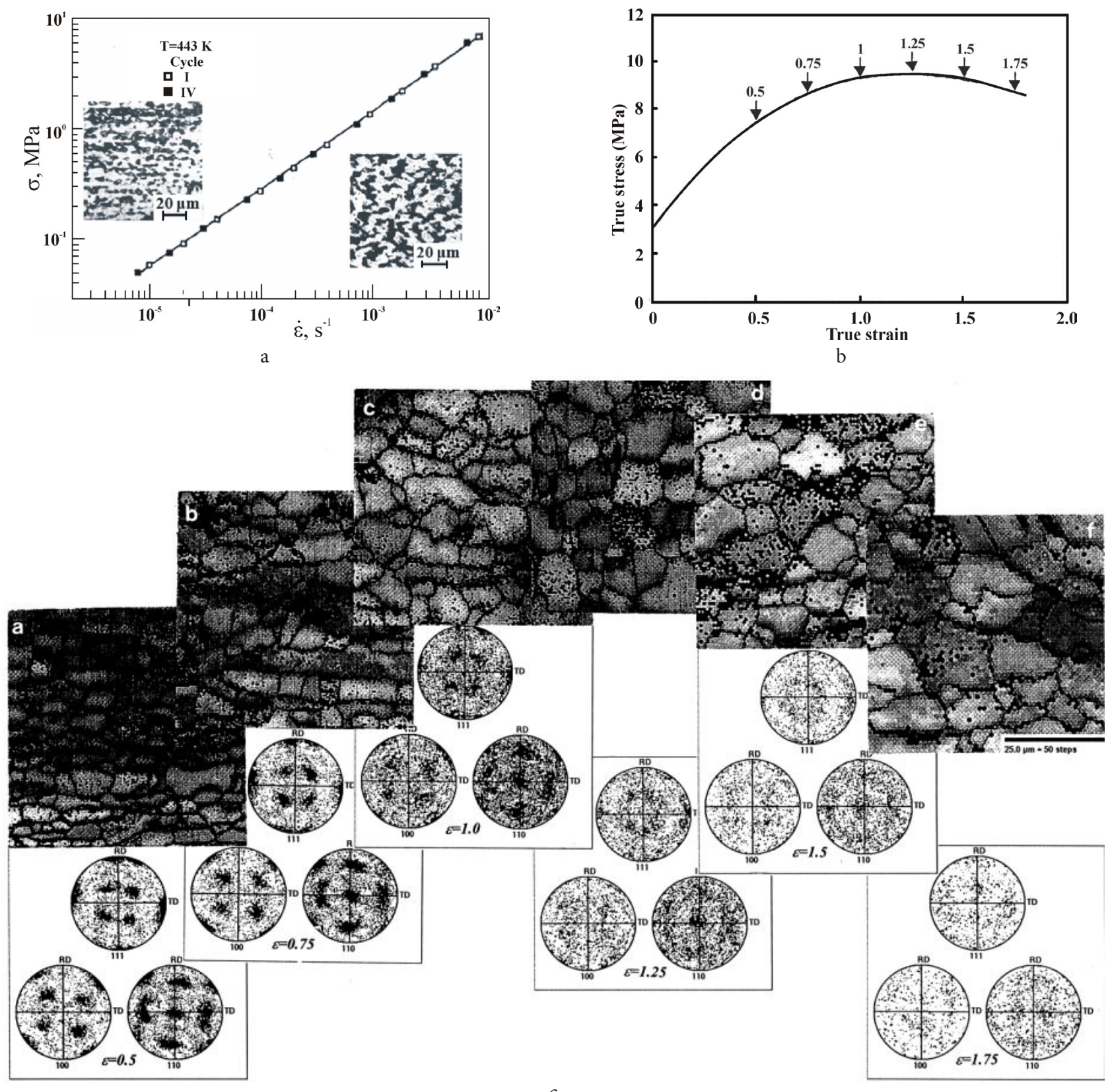

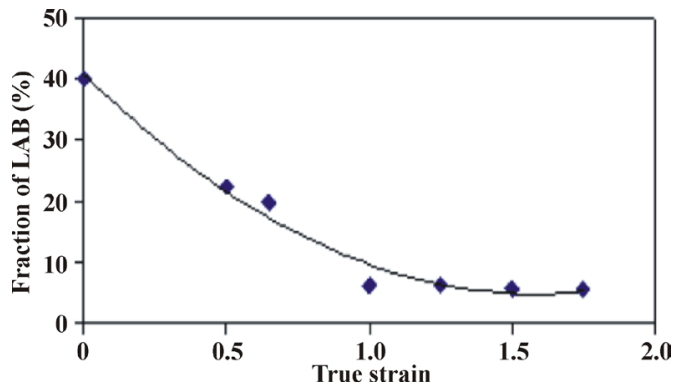

d

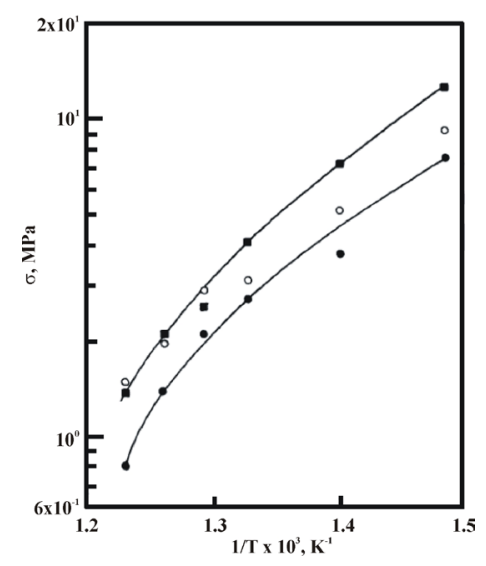

e

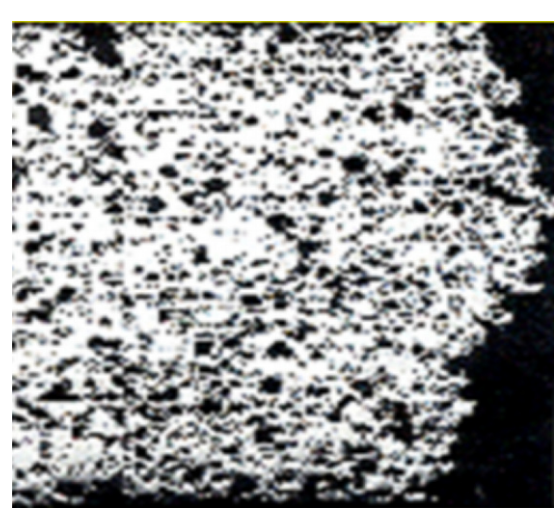

f

Fig. 4. Concurrent microstructure evolution influencing the nature of stress-strain and stress-strain rate plots: pseudo-steady state stressstrain rate plot for $\mathrm{Pb}-\mathrm{Sn}$ eutectic as caused by equal and opposite effects of grain growth and elongated grains changing into equiaxed grains [10] (a); nature of stress-strain curve of 8090 Al-Li alloy (b) with concurrent evolution in microstructure and texture (c), and misorientation angle changing from low angle boundaries to high angle boundaries [16] (d); contribution of concurrent grain growth to flow stress as a function of test temperature in an Al-Cu eutectic [9] (e); an example of extensive cavity formation in IN718 superalloy during superplastic deformation (f). 
by dynamic recovery. Here, the flow stress increases due to increase in dislocation density but, at the same time, some of the dislocations getting generated also get annihilated, the way it happens in conventional high temperature deformation [18]. Shown in Fig. 4e is the plot of flow stresses obtained in an $\mathrm{Al}-\mathrm{Cu}$ eutectic alloy as a function of inverse absolute temperature at the onset of plastic strain $(\varepsilon=0)$ and $\varepsilon=0.30$. The flow stress was also obtained by measuring the grain size at $\varepsilon=0.30$ at different test temperatures and calculating the associated contribution by making use of constitutive relationship between grain size and flow stress. As seen in the figure, the experimental and calculated values of flow stress are comparable at higher test temperatures but there occurs increasing deviation between the two with decreasing temperature. Another microstructure feature during superplastic deformation is the occurrence of cavitation [1]. Shown in Fig. $4 \mathrm{f}$ is one such example in an IN718 superalloy in spite of which the superplastic elongation of $485 \%$ was obtained in this material deformed at temperature of $1198 \mathrm{~K}$ and strain rate of $10^{-4} \mathrm{~s}^{-1}$ [13]. The formation of cavities causes weakening, which results in flow softening. However, the advanced stage of cavitation can cause flow hardening instead of flow softening. In fact, excessive cavitation creates a large number of cavities but leaving un-cavitated ligaments between the set of two adjacent cavities. These ligaments act as micro-tensile specimens within the macroscopic sample, which is subjected to constant speed or strain rate deformation externally but in reality or effectively this sample locally deforms at much higher strain rates through the contributions to deformation by several ligaments inside the macro-sample. Since superplasic material is highly strain rate sensitive, the flow stress correspondingly increases with increasing strain prior to fracture. Thus, the sinusoidal nature of stress-strain curve in Fig. $3 \mathrm{~d}$ can be explained in the sequence of (i) increase in flow stress due to grain growth, (ii) decrease in flow stress due to cavitation and (iii) further increase in flow stress with strain due to deformation of inter-cavity ligaments [14].

\section{Experimental and analytical approaches realized for constitutive relationship}

If the assumptions of constitutive relationship for steady state, in terms of the existence of stable microstructure as well as no variation in stress with strain, are met then it is easy to consider the stress at plateau and the stable microstructure to obtain the experimental parameters of the constitutive relationship. For this, tensile or compression tests are commonly employed either by constant strain rate or differential (varying) strain rate test techniques. Also can be used are the creep tests or stress relaxation tests for the data required at much lower end of the stress-strain rate plot. In the event of deviation from these conditions of steady state, attempts are generally made either to eliminate the transient behavior of flow curves prior to collecting the stress-strain rate data or minimize the strain level and microstructure variation for obtaining the stress-strain rate data. The same is presented below from the literature by describing their limitations. (i) The peak stress values from the stress-strain curves at constant strain rates and temperatures are collected for selected grain sizes. Since the strains to peak stress are generally low it is assumed that the initial microstructure remains unchanged up to peak stress. However, it is recognized that the microstructure change is rapid in the beginning of deformation in which case the peak stresses can be affected by selected strain rate and temperature of deformation.

(ii) Since the peak stress is followed by either flow softening and/or pseudo-steady state, all the samples to be deformed for collecting the stress-strain rate data are subjected to prior straining, to some fixed strain value, at the common strain rate and temperature condition. Doing this reasonably eliminates subsequent non-steady state as well as transient flow behavior from the stress-strain curves. Fig. 5a shows such stress-strain rate plot for $\mathrm{Pb}-\mathrm{Sn}$ eutectic alloy which had undergone prior straining of $30 \%$ in tension at $421 \mathrm{~K}[19]$.

(iii) Limiting the tests to minimum strain at each strain rate can be done by differential strain rate tests. In spite of the popularity of differential strain rate tests, there appeared several methods of data analysis for getting the correct stress values between adjacent strain rates applied for obtaining $m$ values [20]. Nevertheless, no true steady state appears unless prior straining as in (ii) above is given. In the case of flow stress variation with strain through concurrent microstructure changes, it was recently suggested to get the average value of $m$ by making use of the differential strain rate test technique in the forward strain rate change test followed by a reverse strain rate change test. This is illustrated in Fig. 5b for a near $\alpha$-Ti alloy [21].

(iv) If the microstructure change that occurs is concurrent grain growth alone, then the stress value can be corrected for the same for getting more reliable values of the parameters of constitutive relationship. However, flow hardening as shown in Fig. $4 \mathrm{e}$ is also partly contributed by conventional work hardening in certain cases and makes the correction based on grain size less realistic [9].

(v) In general, processing of superplastic materials leads to a wide variation in the microstructure produced. Such microstructures also undergo further changes in the course of superplastic deformation, which results in variation in stress with strain. Consequently, the parameters of the constitutive relationship themselves may vary with strain as shown in Fig. 5c, d for $m$ and $Q$, respectively [22]. Such variation can be brought by introducing the strain effect in the constitutive relationship. However, this approach leaves us with the problem of understanding the role of microstructure in the stress-strain rate relationship.

(vi) In view of the change in microstructure and variation in flow stress with strain, it was suggested to get the stress-strain rate data for steady state by using prior straining of samples with stable equiaxed grains. Then, by taking this as a reference stress-strain rate plot, one can plot such curves for non-equiaxed microstructures. A comparison of the stressstrain rate plots for equiaxed grains, banded microstructure and elongated microstructure are made in Fig. 5e. Such comparison can bring the effect of grain shape in the nature of stress-strain rate curves [23]. 

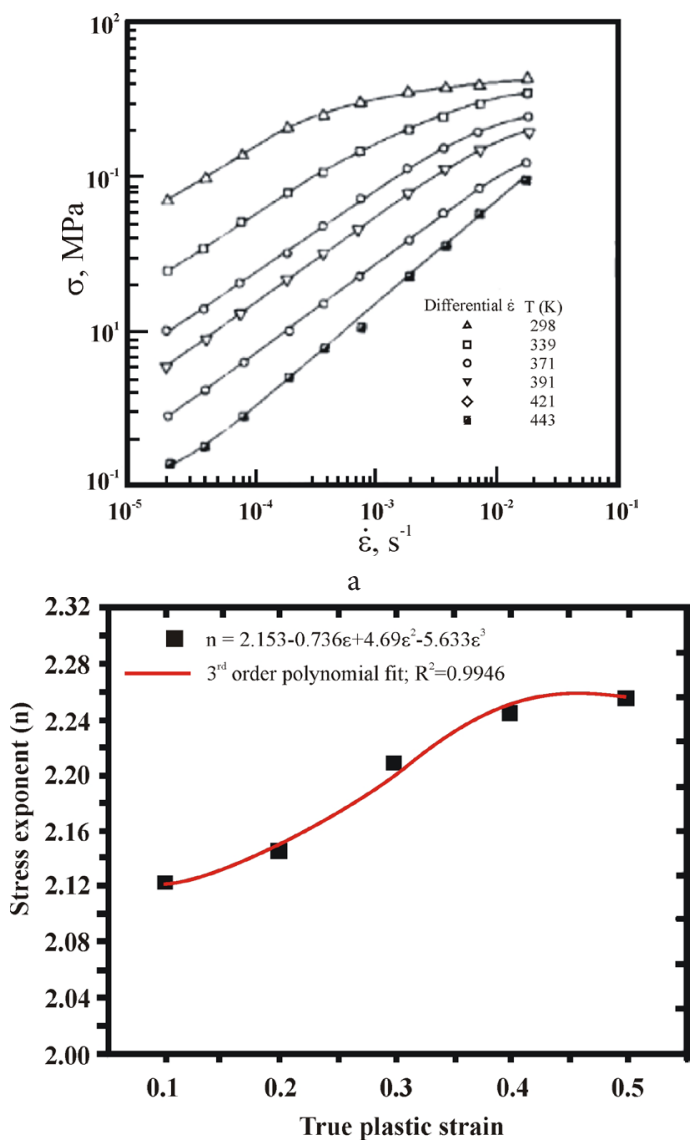

c

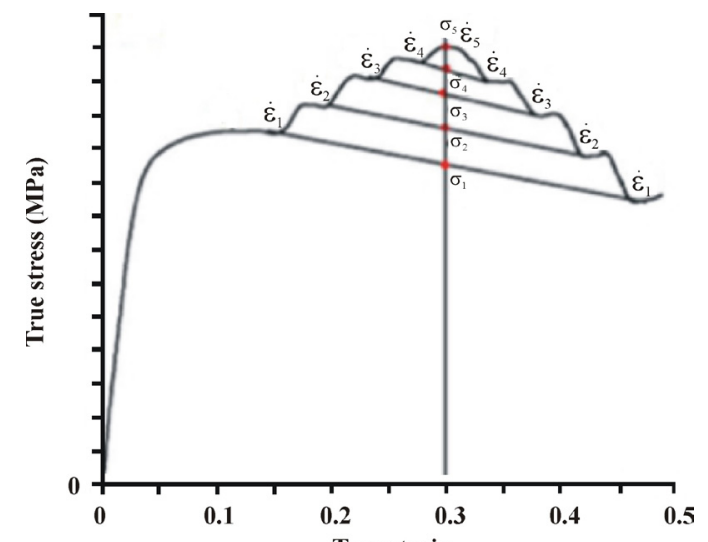

$\mathrm{b}$

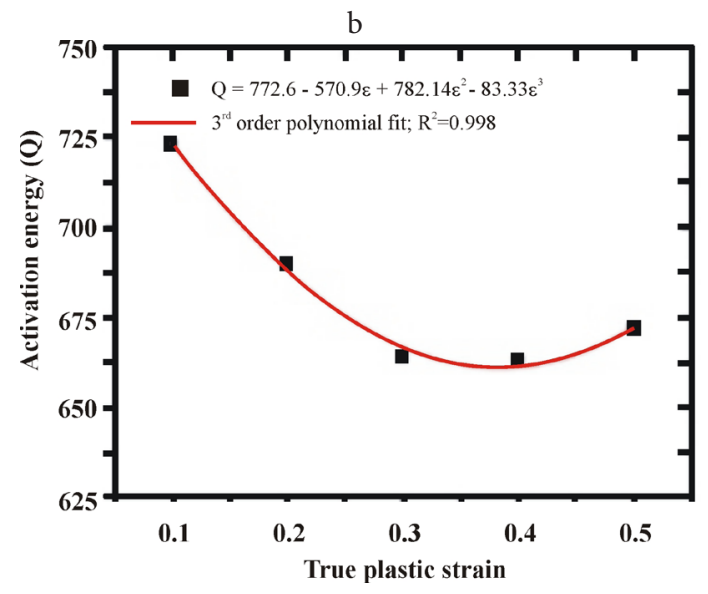

d

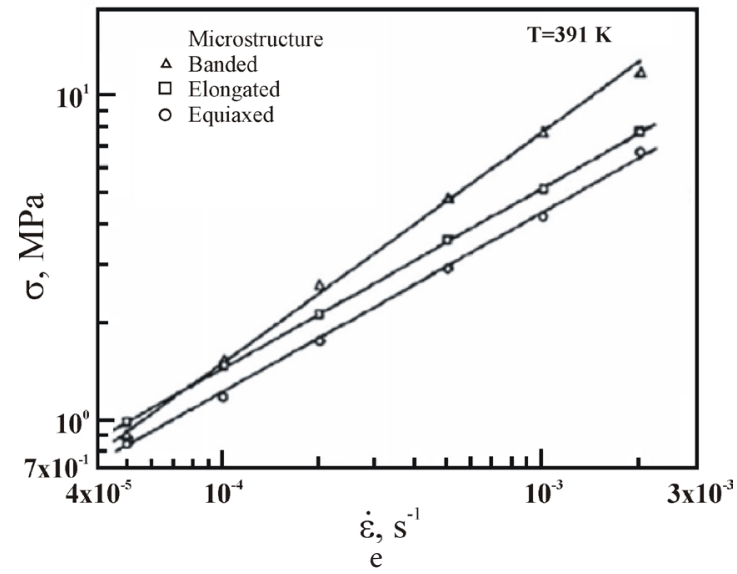

Fig. 5. Approaches adopted to minimize the deviation from steady state towards evaluating the parameters of experimental constitutive relationship: use of suitable prior straining to eliminate the early transient flow in Pb-Sn eutectic [19] (a), schematic of differential strain rate test repeated in the forward strain rate and reverse strain rate change sequence and taking the mean stress at the common strain rates [21] (b), variation in the values of parameters $m$ and $Q$ with strain in near $\alpha$-Ti alloy IMI 834 [22] (c,d), making use of the stress-strain rate plot for the stabilized microstructure as an standard and evaluating the relative contributions of non-equiaxed microstructure on flow stress in $\mathrm{Pb}-\mathrm{Sn}$ eutectic [23] (e).

\section{Summary and concluding remarks}

While the fine equiaxed and stable microstructure is a prime requirement for the materials to exhibit superplasticity there remain ample evidences that the initial non-equiaxed grains can undergo rapid change towards equiaxed grains but along with concurrent grain growth, as enhanced by the basic mechanisms of superplastic deformation themselves. For example, grain boundary sliding is the dominant mechanism of superplastic deformation but it does require accommodating processes like diffusion and/or intra- granular slip, with the former seen in the form of grain boundary migration contributing to grain growth. In the absence of sufficient accommodation processes for grain boundary sliding there can occur stress concentration to cause cavitation. The operation of different mechanisms for superplastic deformation encounters obstacles the effect of which is found in the form of threshold and/or internal stress as a major fraction of applied stress. Several mechanisms for superplastic deformation are proposed in the literature but a realistic approach to account for the wide range of deviations needs to consider the variation in 
flow stress with strain through the microstructure change or an approach towards pseudo-steady state condition for major part of superplastic deformation. To bridge the gap between the ideal case of steady-state for superplasticity and the realistic case of deviation from this, the parameters of constitutive relationship should include the instantaneous microstructure and consider effective stress for deformation instead of applied stress.

\section{References}

1. B. P. Kashyap, A. K. Mukherjee. Res. Mechanica. 17, 293 (1986).

2. M. Suery, B. Baudelet. Rev. Phys. Appl. 13, 58 (1978).

3. B. P. Kashyap, A. Arieli, A. K. Mukherjee. J. Mater. Sci. 20, 2661 (1985).

4. O.D. Sherby, J. Wadsworth. Prog. Mater. Sci. 33, 169 (1989).

5. M. G. Zelin, A. K. Mukherjee. Mater. Sci. Eng. A208, 210 (1996).

6. A. K. Mukherjee, J. E. Bird, J. E. Dorn. Trans. Am. Soc. Met. 62, 155 (1969).

7. J. Cadek. Creep in Metallic Materials. Amsterdam, Elsevier Sci. Publ. (1988) 372 p.

8. B. P. Kashyap, G. S. Murty. Trans. JIM. 22, 515 (1981).

9. B. P. Kashyap, K. Tangri. Metall. Trans. A. 18A, 417 (1987).

10. B.P. Kashyap, G.S. Murty. Metall. Trans. A. 13A, 53
(1982).

11. W. Fan, B.P. Kashyap, M.C. Chaturvedi. Mater. Sci. Techno. 17, 439 (2001).

12. B.P. Kashyap, A. K. Mukherjee. Metall. Trans. A. 14A, 1875 (1983).

13. B. P. Kashyap, M. C. Chaturvedi. Mater. Sci. Techno. 16, 147 (2000).

14. B. P. Kashyap, M. C. Chaturvedi. Mater. Sci. Techno. 19, 1369 (2003).

15. B.P. Kashyap, M.C. Chaturvedi. Unpublished Work (2000).

16. B. P. Kashyap, M. C. Chaturvedi. Mater. Sci. Techno. 19, 1369 (2003).

17. B. P. Kashyap, W. Fan, M.C. Chaturvedi. Mater. Sci. Techno. 17, 237 (2001).

18. T.H. Courtney, Mechanical Behavior of Materials. Long Grove, Waveland Press Inc. (2005) 733 p.

19. B. P. Kashyap, G.S. Murty. Mater. Sci. Engg. 50, 205 (1981).

20. K. A. Padmanabhan, G. J. Davis. Superplasticity. Berlin, Springer-Verlag (1980) 312 p.

21. S. M. Jagadeesh Babu, B. P. Kashyap, N. Prabhu, R. Kapoor, R. N. Singh, J. K. Chakravartty. Mater. Sci. Engg. A. 679, 75 (2017).

22. I. Balasundar, T. Raghu, B. P. Kashyap. Prog. Natural Sci. Mater. Intl. 23, 598 (2013).

23. B. P. Kashyap, G. S. Murty. J. Mater. Sci. 18, 2063 (1983). 